GA-A23495

\title{
PROGRESS IN QUANTIFYING THE EDGE PHYSICS OF THE H-MODE REGIME IN DIII-D
}

\author{
by \\ R.J. GROEBNER, D.R. BAKER, J.A. BOEDO, K.H. BURRELL, \\ T.N. CARLSTROM, R.D. DERANIAN, E.J. DOYLE, J.R. FERRON, P. GOHIL, \\ G.R. MCKEE, R.A. MOYER, C.L. RETTIG, T.L. RHODES, D.M. THOMAS, \\ T.H. OSBORNE, and W.P. WEST
}




\section{DISCLAIMER}

This report was prepared as an account of work sponsored by an agency of the United States Government. Neither the United States Government nor any agency thereof, nor any of their employees, makes any warranty, express or implied, or assumes any legal liability or responsibility for the accuracy, completeness, or usefulness of any information, apparatus, product, or process disclosed, or represents that its use would not infringe privately owned rights. Reference herein to any specific commercial product, process, or service by trade name, trademark, manufacturer, or otherwise, does not necessarily constitute or imply its endorsement, recommendation, or favoring by the United States Government or any agency thereof. The views and opinions of authors expressed herein do not necessarily state or reflect those of the United States Government or any agency thereof. 
GA-A23495

\title{
PROGRESS IN QUANTIFYING THE EDGE PHYSICS OF THE H-MODE REGIME IN DIII-D
}

\author{
by \\ R.J. GROEBNER, D.R. BAKER, J.A. BOEDO, † K.H. BURRELL, \\ T.N. CARLSTROM, R.D. DERANIAN, E.J. DOYLE, $\diamond$ J.R. FERRON, P. GOHIL, \\ G.R. MCKEE, $\triangle$ R.A. MOYER, † C.L. RETTIG, $\diamond$ T.L. RHODES, $\diamond$ D.M. THOMAS, \\ T.H. OSBORNE, and W.P. WEST
}

This is a preprint of a paper presented at the 18th IAEA Fusion Energy Conference, October 4-10, 2000 in Sorrento, Italy and to be published in the Proceedings.

\author{
†University of California, San Diego, California \\ ¥Cardiff University, Wales, United Kingdom \\ $\checkmark$ University of California, Los Angeles, California \\ $\Delta$ University of Wisconsin, Madison, Wisconsin
}

\begin{abstract}
Work supported by
the U.S. Department of Energy under Contract DE-AC03-99ER54463, and Grants DE-FG03-95ER54294, DE-FG03-86ER53225, and DE-FG03-96ER54373
\end{abstract}




\title{
Progress in Quantifying the Edge Physics of the H-mode Regime in DIII-D
}

\author{
D.M. Thomas, ${ }^{1}$ T.H. Osborne, ${ }^{1}$ and W.P. West ${ }^{1}$ \\ ${ }^{1}$ General Atomics, P.O. Box 85608, San Diego, California 92186-5608 \\ email: groebner@fusion.gat.com \\ 2University of California, San Diego, California \\ ${ }^{3}$ Cardiff University, Wales, United Kingdom \\ ${ }^{4}$ University of California, Los Angeles, California \\ 5 University of Wisconsin, Madison, Wisconsin
}

R.J. Groebner, ${ }^{1}$ D.R. Baker, ${ }^{1}$ J.A. Boedo, ${ }^{2}$ K.H. Burrell, ${ }^{1}$ T.N. Carlstrom, ${ }^{1}$ R.D. Deranian, ${ }^{3}$ E.J. Doyle, ${ }^{4}$ J.R. Ferron, ${ }^{1}$ P. Gohil, ${ }^{1}$ G.R. McKee, ${ }^{5}$ R.A. Moyer, ${ }^{2}$ C.L. Rettig, ${ }^{4}$ T.L. Rhodes, ${ }^{4}$

\begin{abstract}
Edge conditions in DIII-D are being quantified in order to provide insight into the physics of the $\mathrm{H}$-mode regime. Electron temperature is not the key parameter that controls the $\mathrm{L}-\mathrm{H}$ transition. Gradients of edge temperature and pressure are much more promising candidates for such parameters. The quality of $\mathrm{H}-$ mode confinement is strongly correlated with the height of the $\mathrm{H}$-mode pedestal for the pressure. The gradient of the pressure appears to be controlled by MHD modes, in particular by kink-ballooning modes with finite mode number $n$. For a wide variety of discharges, the width of the barrier is well described with a relationship that is proportional to $\left(\beta_{\mathrm{p}}^{\text {ped }}\right)^{1 / 2}$. An attractive regime of confinement has been discovered which provides steady-state operation with no ELMs, low impurity content and normal H-mode confinement. A coherent edge MHD-mode evidently provides adequate particle transport to control the plasma density and impurity content while permitting the pressure pedestal to remain almost identical to that observed in ELMing discharges.
\end{abstract}

\section{Introduction}

The ELMing $\mathrm{H}$-mode discharge is the baseline scenario for a next-step tokamak device. The characteristics of these discharges are strongly affected by boundary conditions near the separatrix. In particular, 1) access to the $\mathrm{H}$-mode state is controlled by boundary physics, 2) the amount of the confinement enhancement of the $\mathrm{H}$-mode plasma depends on the height of the $\mathrm{H}$-mode transport barrier and 3) control of the plasma density and impurity content is obtained by transport processes at the plasma periphery. The edge physics is not well enough understood to permit reliable predictions from theory-based models as to whether the $\mathrm{H}$-mode state will be attained and as to what level of confinement will be achieved in the $\mathrm{H}$-mode state. This lack of knowledge is one of the greatest impediments for the design of future machines. Moreover, acceptable particle control is normally achieved with ELMs. Unfortunately, ELMs have the deleterious effect of producing large transient heat pulses on the divertor plates, which may lead to unacceptably fast erosion of the plates.

Part of the DIII-D program is directed at identifying and quantifying the edge physics relevant to $\mathrm{H}$-mode discharges. Recent work shows that the $\mathrm{H}$-mode transition is not controlled by the electron temperature alone. However, edge gradients of temperature and pressure are candidates for ingredients in the causal chain of physics that causes the transition. New studies provide additional evidence that the height of the $\mathrm{H}$-mode pressure pedestal has a profound effect on the value of the plasma's energy confinement. Confinement improvements with increasing triangularity are strongly correlated with increases in the height of the pedestal, which in turn are due primarily to improved MHD stability and therefore to higher edge pressure gradients. A remarkable operating regime, the "quiescent H-mode", has been discovered. This regime provides steady state operation with no ELMs, good particle control and standard $\mathrm{H}$-mode confinement. The key to this mode of operation is the existence of a continuous edge MHD mode which evidently provides enough transport to exhaust plasma particles but does not significantly modify the edge pressure gradient from that obtained in normal ELMing discharges.

\section{Edge Conditions for $\mathrm{H}$-mode Access}

Attainment of a "critical" edge electron temperature has been proposed as a threshold criterion for the L-H transition [1]. On DIII-D, several lines of research indicate that this is 
not the case. First, operational space diagrams of $T_{e}$ and $n_{e}$ show that there is a very significant overlap of $\mathrm{T}_{\mathrm{e}}$ values from the $\mathrm{L}$-mode and $\mathrm{H}$-mode states $[2,3]$. There is no single value of $\mathrm{T}_{\mathrm{e}}$ which can be considered as a "critical" value for the transition. These data have been evaluated where the edge density gradient has its maximum value. This location is typically less than $1 \mathrm{~cm}$ inboard of the separatrix at the outer midplane, is in the region which becomes the $\mathrm{H}$-mode transport barrier and is an ideal location for evaluating edge parameters of relevance to the $\mathrm{L}-\mathrm{H}$ transition

Studies of the $\nabla B$ drift effect are a second line of research which show that the condition for the $\mathrm{H}$-mode transition depends on more physics than just the edge fluid parameters, density, temperature and pressure [4]. Discharges have been prepared with both signs of the toroidal magnetic field $\mathrm{B}_{\mathrm{T}}$ for which the edge fluid parameters were nearly identical. However, for one sign of the magnetic field, the heating power was within $10 \%-30 \%$ of the $\mathrm{H}$-mode threshold while for the other sign of $\mathrm{B}_{\mathrm{T}}$, the same amount of power was a factor of 3-5 below the threshold. Thus, the condition for obtaining the $\mathrm{H}$-mode is not just the requirement that a critical value of the edge temperature or pressure is achieved.

The most compelling evidence that there is not a critical $\mathrm{T}_{\mathrm{e}}$ for the transition is provided by experiments in which deuterium pellets have induced transitions to $\mathrm{H}$-mode (PIH-mode) when the heating power was below the nominal $\mathrm{H}$-mode threshold [5]. In these discharges, both the edge $\mathrm{T}_{\mathrm{e}}$ and $\mathrm{T}_{\mathrm{i}}$ were reduced by $\approx 50 \%$ in the $\mathrm{L}$-mode by injection of a pellet (Fig. 1). Nevertheless, the $\mathrm{H}$-mode transition occurred within a few milliseconds after pellet injection. These results are inconsistent with the idea that the $\mathrm{H}$-mode transition occurs when a "critical" edge temperature is achieved.

Pattern recognition algorithms have been developed and used to search for operational space diagrams that best classify the $\mathrm{L}$-mode and $\mathrm{H}$-mode states [6-7]. Results from this research show that parameters based on $\nabla \mathrm{T}_{\mathrm{e}}$ or $\nabla \mathrm{P}_{\mathrm{e}}$, measured at the location of steepest edge density as described above, do a very good job of distinguishing the L- and H-states. It is not surprising that such a parameterization should work because increases in the edge gradients are part of the L-H transition phenomenology. However, it is surprising in a quantitative sense how well these parameterizations distinguish between the L- and $\mathrm{H}$-states. For example, in Fig. 2, which shows an operational space diagram of $\nabla \mathrm{T}_{\mathrm{e}} / \mathrm{T}_{\mathrm{e}} \mathrm{vs} . \nabla \mathrm{P}_{\mathrm{e}} / \mathrm{n}_{\mathrm{e}}$, it is possible to draw a line that divides the two states nicely. This work has motivated studies which show that gradients of electron and ion temperature and pressure, at the very periphery of the plasma, systematically increase in the L-phase of discharges which make a transition to $\mathrm{H}$-mode, as illustrated in Fig. 3. These changes are larger and more consistently observed than changes in the edge temperatures or pressures [3] and suggest that increases of edge gradients may be part of the causal chain of events for the L-H transition. Changes in these gradients might stimulate the transition by modifying the edge electric field shear in L-mode.
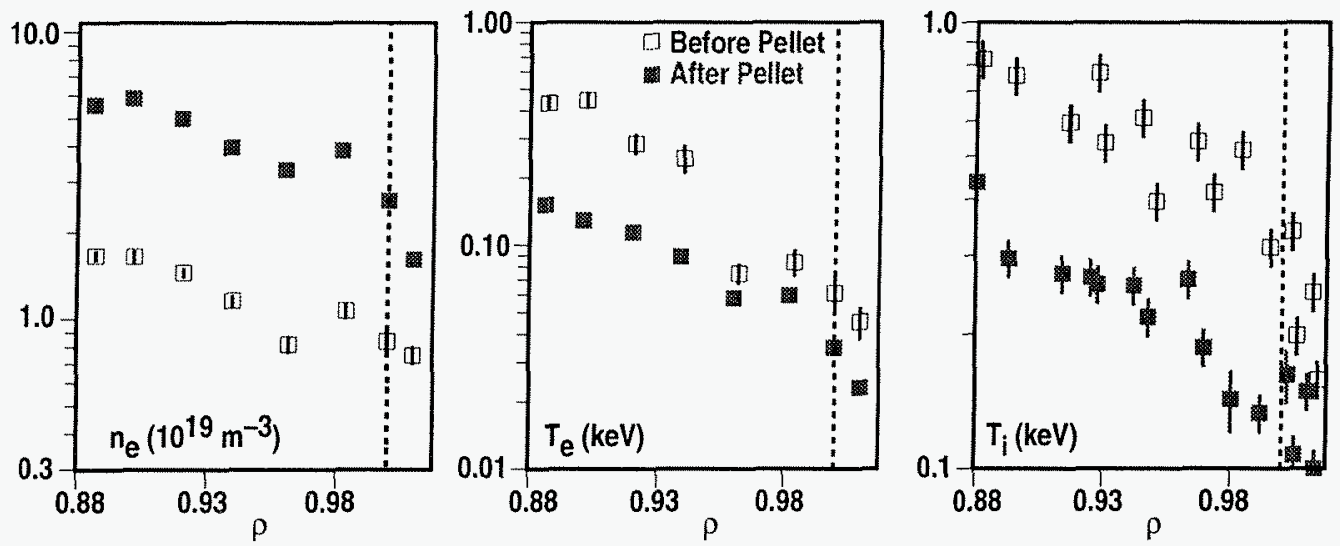

FIG. 1 . Edge $n_{e}$ rises by about factor of 3 and edge $T_{e}$ and $T_{i}$ decrease by about $50 \%$ from before pellet injection ( $18 \mathrm{~ms}$ before $H$-mode transition) to after pellet injection ( $6 \mathrm{~ms}$ before $H$-mode transition). Separatrix shown by vertical dashed lines. 


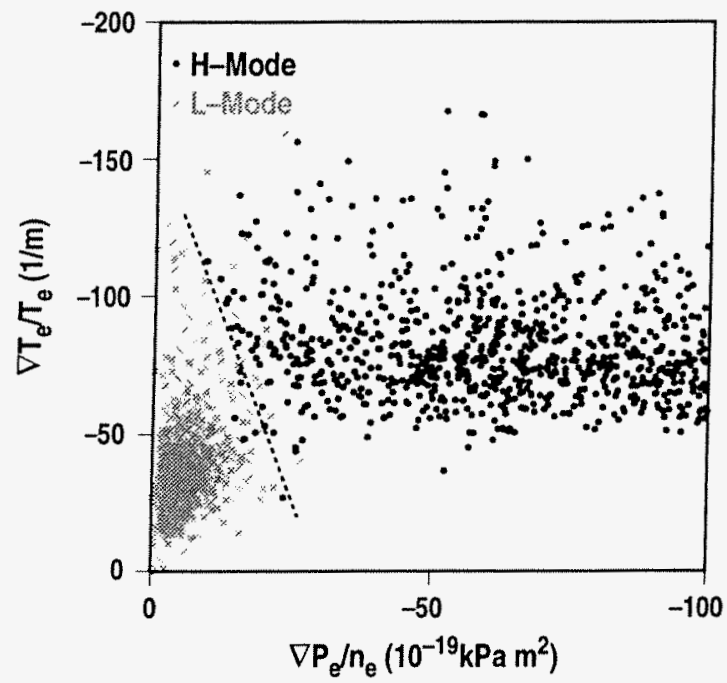

FIG. 2. Operational space diagram showing that $\nabla T_{e} / T_{e}$ and $\nabla P_{e} / n_{e}$ distinguish very well between $L$-mode and $H$-mode. Data are drawn from scans of density, current and toroidal field and from discharges with PIH-modes. Dashed line shows approximate division between the two states. Of the $2000+$ points used for the figure, more than $99 \%$ are classified correctly by the dashed line.

\section{Pedestal Scaling}

Previous studies have shown that the confinement of $\mathrm{H}$-mode plasmas increases as the height of the pressure pedestal $P_{\text {ped }}$ increases [8]. More recent results from DIII-D show that this conclusion is valid for a wider range of conditions than were covered in the original studies. For example, the confinement degradation of high density

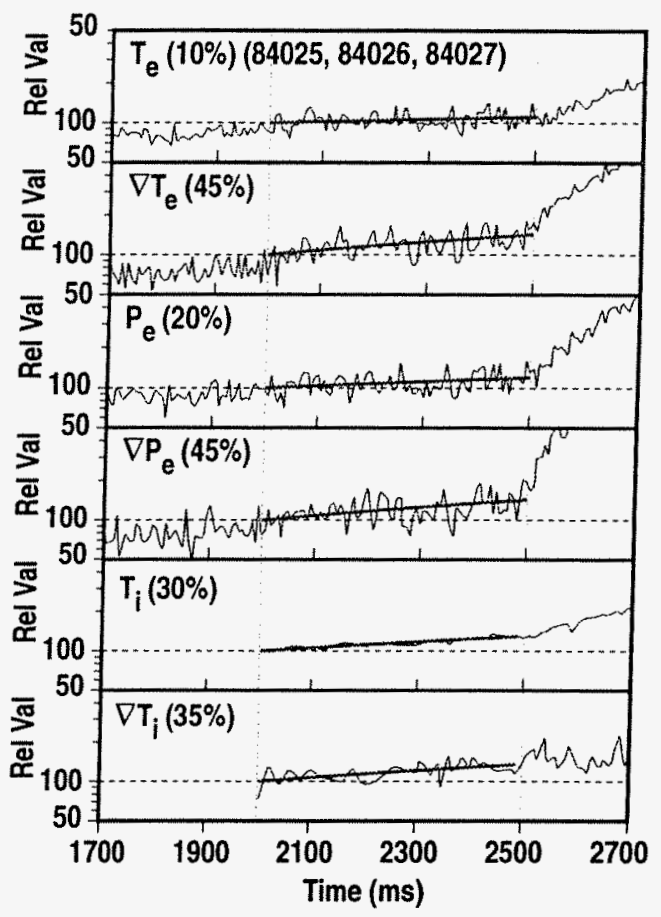

FIG. 3. Thin lines show time-series for $T_{e}, \nabla T_{e}$, $P_{e}$ and $\nabla P_{e}$, evaluated at location of largest $n_{e}$ gradient, and $T_{i}$ and $\nabla T_{i}$ evaluated at the largest $C$ VI density gradient, for average of three identical discharges with a low H-mode power threshold (1.5 MW). Thick lines show linear fits to the data for the L-phase, delineated by vertical dashed lines. The data are plotted as relative values (percentages) of the initial value of the linear fit. Percentage changes in each parameter during the L-phase, as determined from the linear fits, are also shown. The largest changes in $L$-mode occurred for $\nabla T_{i}, \nabla T_{e}$ and $\nabla P_{e}$, which increased by $35 \%-45 \%$.

discharges during gas fueling is strongly correlated with a reduction of $T_{\text {ped }}$ and in $P_{\text {ped }}[9]$. Also, confinement improvements with increased triangularity are correlated with increases of $\mathrm{P}_{\text {ped }}[10]$. Thus, predicting the core $\mathrm{H}$-mode confinement in future devices requires knowledge of the values of pressure or of temperature and density at the $\mathrm{H}$-mode pedestal.

Studies of pedestal scaling have been divided into studies of the electron pressure gradient and of $\Delta_{\mathrm{pe}}$, the width of the transport barrier for $\mathrm{P}_{\mathrm{e}}$. The pressure gradient is controlled by MHD stability. Although there are discharges in which the measured gradients are consistent with limits expected for infinite-n ideal ballooning modes [11], there are many conditions in DIII-D in which the gradients substantially exceed these predictions. Calculations and modeling of experimental data show that for appropriately shaped discharges, the bootstrap current driven by the edge pressure gradient can provide stability against infinite-n ballooning modes and allow the plasma access to the second stable region [12]. Recent results indicate that for these plasmas, the pressure gradients are limited by finite- $n$ kink/ballooning modes $[11,13]$.

The previous work has shown that the pressure barrier width $\Delta_{\text {pe }}$ shows a rather small variation in DIII-D for a wide range of discharge characteristics. The best scaling which has been determined shows that $\Delta_{\mathrm{pe}}$ is proportional to $\left(\beta_{\mathrm{p}}^{\mathrm{ped}}\right)^{1 / 2}$, where $\beta_{\mathrm{p}}^{\text {ped }}$ is beta-poloidal for the electrons, evaluated on the pedestal [8]. Determined three years ago, this scaling remains the best description of a wide range of DIII-D discharges. Experiments with pumping which broke the connection of density and temperature showed that $\Delta_{\text {pe }}$ does not scale with the ion poloidal gyroradius [14]. 
As noted above, confinement improvements that occur with increases in triangularity $\delta$ are correlated with increases in $\mathrm{P}_{\text {ped }}$. In these studies, the height of the $\mathrm{H}$-mode pressure pedestal increased by a factor of two as the upper $\delta$ was increased from 0.0 to 0.5 . This increase was due primarily to an increase in the pressure gradient [10]. All discharges were in the second stable region for infinite- $n$ ballooning modes. Good agreement has been obtained between the measured pressure gradient and calculations based on medium-n kink/ballooning modes [11]. In contrast, the barrier width increased weakly with $\delta$ and was described reasonably well by the scaling relationship based on $\left(\beta_{\mathrm{p}}^{\text {ped }}\right)^{1 / 2}$ [10]. In brief, increasing $\delta$ leads to improved MHD stability at the edge which in turn allows for a higher pressure pedestal and therefore higher stored energy.

\section{Quiescent H-mode}

A remarkable mode of steady-state $\mathrm{H}$-mode operation has been discovered which has several attractive features from the point of view of a reactor. This mode, called the "quiescent $\mathrm{H}$-mode" has been obtained for a range of conditions with counter-injected neutral beams and cryopumping to lower the edge density. It has standard $\mathrm{H}$-mode confinement with no ELMs and therefore no pulsed heat load to the divertor targets. The salient characteristics of a typical discharge are shown in Fig. 4. After a brief period of ELMing, the discharge entered a steadystate phase, free of ELMs, which lasted for $3.5 \mathrm{~s}$, limited only by machine power supplies. During the steady-state phase, the density remained constant, the confinement time remained constant at about twice the ITER-89P level, the radiated power remained constant at about $30 \%$ of the input power, the impurity content remained constant with core Zeff of about 2 and the edge pressure gradient remained constant at the same levels seen during the ELMing phase. These observations are in marked contrast to the usual $\mathrm{H}$-mode phenomenology in which impurity levels and radiation rapidly increase when ELMs are absent.

These discharges are called "quiescent" rather than "ELM-free", because the term "ELMfree" usually refers to that phase of an $\mathrm{H}$-mode discharge in which the pressure gradient is increasing to the value which causes ELMs. In marked contrast, these discharges have pressure gradients that are at the limit that can cause ELMs, but ELMs are not observed. Figure 5 shows a comparison of edge $T_{e}, n_{e}, p_{e}$ and $T_{i}$ profiles from the ELMing and quiescent phases of a typical discharge. With the exception that $T_{i}$ is higher in the quiescent phase, the two sets of profiles are very similar and allow for the confinement of the quiescent phase to be as good as that of the ELMing phase.

Despite the lack of ELMs, the quiescent phase is accompanied by low amplitude coherent oscillations observed both on magnetic diagnostics (Fig. 4) and on diagnostics sensitive to density fluctuations. These oscillations exhibit several harmonics, typically in the range of $n=1-9$, and may be due to the presence of a magnetic island. Density fluctuation diagnostics indicate that the strength of this "multi-harmonic mode" (MHM) is strongest in the vicinity of the $\mathrm{H}$-mode transport barrier, although signatures of the mode are seen deeper into the core of the plasma. Although the MHM is of interest because of its unique characteristics, it appears to have the practical effect of causing enough particle transport at the edge to provide 
plasma density control and to maintain plasma cleanliness. More work is needed to identify the MHM.

It is not clear what physics allows the MHM rather than ELMs to exist at the plasma edge. One possibility is that the shape of the radial electric field, obtained with reversed current, modifies the stability of ELMs. These discharges have very desirable characteristics from the point of view of reactor operation. They have standard $\mathrm{H}$-mode confinement but no pulsed heat load to the divertor, no impurity accumulation and appear able to run indefinitely.

\section{Acknowledgment}

Work supported by U.S. Department of Energy under Contract DE-AC0399ER54463 and Grants DE-FG0395ER54294, DE-FG03-86ER53225 and DEFG03-96ER54373.

\section{References}

[1] IGITKHANOV, Y., et al., Plasma Phys. Control Fusion 40, 837 (1998).

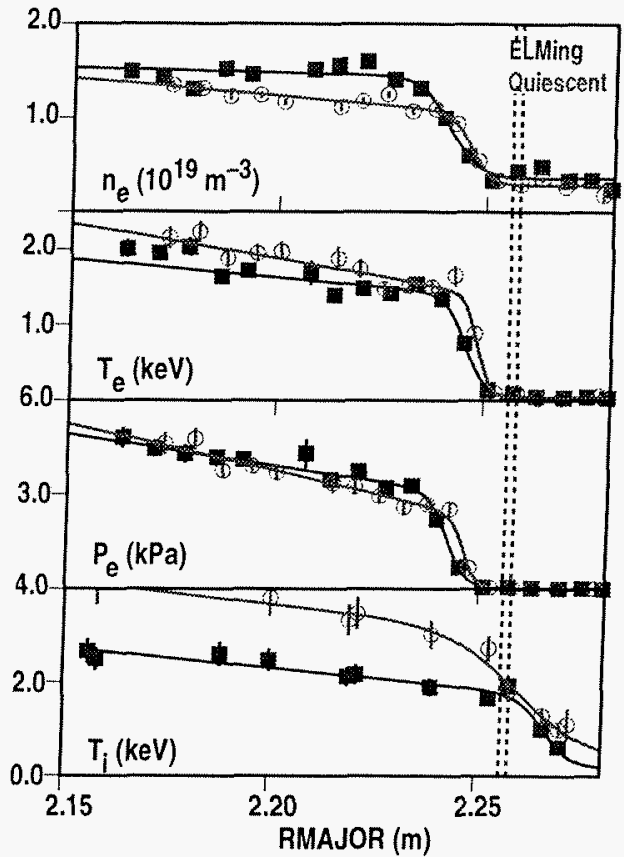

FIG. 5. Typical edge profiles for $n_{e}, T_{e}$ and $p_{e}$ for ELMing and quiescent phases of same discharge are similar. In particular, gradients in transport barrier are similar. $T_{i}$ is higher in quiescent phase. Vertical dashed lines show locations of separatrices at two times.

[2] CARLSTROM, T.N., et al., Nucl. Fusion. 39, 1941 (1999).

[3] GROEBNER, R.J., et al., "Edge Gradients as Catalysts of the Spontaneous High-mode Transition," to be submitted to Physics of Plasmas.

[4] CARLSTROM, T.N, et al., Proc. of 27th Euro. Conf. on Controlled Fusion and Plasma Physics, Budapest, Hungary, 2000, to be published.

[5] GOHIL, P. et al., submitted to Phys. Rev. Lett.

[6] DERANIAN, R., GROEBNER, R.J., PHAM, D.T., Phys. Plasmas 7, 1235 (2000).

[7] DERANIAN, R., GROEBNER, R.J., PHAM, D.T., "Dynamic Parameter Generation for Classifying Physical Systems," to be submitted to Pattern Recognition.

[8] OSBORNE, T.H., et al., Proc. of the 24th Euro. Conf on Controlled Fusion and Plasma Physics, Berchtesgaden, Germany, 1997 (European Physical Society, Petit-Lancy, 1997), Vol. 21A, p. 1101.

[9] OSBORNE, T.H., et al., Proc. of 14th Int. Conf. on Plasma Surface Interactions, Rosenheim, Germany, 2000, to be published in J. Nucl. Mater.

[10] OSBORNE, T.H., et al., Plasma Phys. Control Fusion 42, A175 (2000).

[11] FERRON, J.R., Phys. Plasmas 7, 1976 (2000).

[12] OSBORNE, T.H., et al., J. Nucl. Mater. 266-9, 131 (1999).

[13] FERRON, J.R., et al., Proc. of 27th Euro. Conf. on Controlled Fusion and Plasma Physics, Budapest, Hungary, 2000, to be published.

[14] OSBORNE, T.H., et al., Plasma Phys. Control. Fusion 40, 845 (1998). 